\title{
A Head-count Measure of Rank Mobility and its Directional Decomposition
}

Citation for published version (APA):

Bossert, W., Can, B., \& D'Ambrosio, C. (2018). A Head-count Measure of Rank Mobility and its Directional Decomposition. Economica, 85(340), 793-807. https://doi.org/10.1111/ecca.12256

Document status and date:

Published: 01/10/2018

DOI:

10.1111/ecca.12256

Document Version:

Publisher's PDF, also known as Version of record

Document license:

Taverne

Please check the document version of this publication:

- A submitted manuscript is the version of the article upon submission and before peer-review. There can be important differences between the submitted version and the official published version of record.

People interested in the research are advised to contact the author for the final version of the publication, or visit the DOI to the publisher's website.

- The final author version and the galley proof are versions of the publication after peer review.

- The final published version features the final layout of the paper including the volume, issue and page numbers.

Link to publication

\footnotetext{
General rights rights.

- You may freely distribute the URL identifying the publication in the public portal. please follow below link for the End User Agreement:

www.umlib.nl/taverne-license

Take down policy

If you believe that this document breaches copyright please contact us at:

repository@maastrichtuniversity.nl

providing details and we will investigate your claim.
}

Copyright and moral rights for the publications made accessible in the public portal are retained by the authors and/or other copyright owners and it is a condition of accessing publications that users recognise and abide by the legal requirements associated with these

- Users may download and print one copy of any publication from the public portal for the purpose of private study or research.

- You may not further distribute the material or use it for any profit-making activity or commercial gain

If the publication is distributed under the terms of Article $25 \mathrm{fa}$ of the Dutch Copyright Act, indicated by the "Taverne" license above, 


\title{
A Head-count Measure of Rank Mobility and its Directional Decomposition
}

\author{
By Walter Bossert†, Burak Cant and Conchita D’Ambrosio $\dagger \dagger$ \\ $\dagger$ University of Montreal \$Maastricht University $\quad$ ††University of Luxembourg
}

Final version received 13 September 2017.

\begin{abstract}
We propose and characterize a new measure of rank mobility. The index is given by the head count of those whose positions change in the move from one period to the next divided by population size. The interpretation of this head-count ratio is straightforward and intuitive. In addition, we illustrate how the measure can be decomposed into an index of upwards mobility and an index of downwards mobility. The axioms used in our characterization results are appealing and easy to justify. We conclude with an application to the Human Development Index, a summary measure of average achievement in key dimensions of human development: a long and healthy life, being knowledgeable and having a decent standard of living.
\end{abstract}

\section{INTRODUCTION}

The measurement of mobility is an issue that is, by now, well-established in the area of economic and social index numbers. The fundamental issue to be addressed is the design of measures that reflect the extent to which members of a society (or, at a more aggregate level, population subgroups or countries) move across social or economic boundaries from one period to the next. A crucial aspect that distinguishes mobility from most other criteria that are used to assess the performance of a society (such as income inequality or poverty) is that mobility is difficult - if not impossible - to define without any reference to intertemporal considerations. Of course, intertemporal approaches to the measurement of inequality, poverty and other social phenomena have been explored, but they can also be defined without any difficulties in a single-period setting; in contrast, there is no mobility without movement. As a consequence, the arguments of a mobility measure are pairs of indicators of economic or social status-one indicator for each of the time periods under consideration.

According to Fields (2008), six aspects of mobility can be identified in the literature. These are time-independence, positional movement, share movement, non-directional income movement, directional income movement, and equalizer of longer-term incomes. See, for instance, Maasoumi (1998), Fields and Ok (1999), and Jäntti and Jenkins (2014) for comprehensive surveys.

While the majority of earlier contributions deal with mobility in the context of income distributions, there has been an increasing interest in the notion of rank mobility - that is, the positional movement of individuals, households or countries in economic or social hierarchies. Rank-based measures are widely applied in empirical research (see, for example, Dickens 1999), but as far as we are aware, only a few contributions, such as D'Agostino and Dardanoni (2009), Cowell and Flachaire (2011) and Bossert et al. (2016), investigate them from a theoretical perspective.

The notion of rank mobility plays a role in Cowell and Flachaire (2011), but its axiomatic analysis is not the focus of that paper. They propose a flexible approach that is based on a general measure of distance between individual statuses. Absolute status levels may or may not be directly observable - much of their notion is based on the status of individuals relative to the position of others. 
Rank correlation measures are nowadays widely used to assess the intergenerational mobility of societies. For example, Chetty et al. (2014) examine the extent of intergenerational mobility in the USA. Using a comprehensive dataset, they show that this type of mobility can vary substantially across different geographical regions. Moreover, they identify variables that correlate with upwards mobility. Venator and Reeves (2015) provide a comparison of rank mobility measures and the more traditional intergenerational elasticity coefficient. In a recent contribution, Nybom and Stuhler (2017) analyze the biases that can be associated with the use of intergenerational elasticities as well as other indicators, including log-linear and rank correlation measures.

Focusing on measures of rank mobility is particularly relevant in the framework of indicators of progress of countries beyond GDP. Notable examples of the latter are the Human Development Index of the United Nations and the more recent Better Life Index of the OECD. What attracts the attention of policymakers and the media is not the value of these composite indicators but the positions of the countries and the changes in the rankings over time. We use the Human Development Index to provide an empirical application of our new measures later in the paper.

D'Agostino and Dardanoni (2009) and Bossert et al. (2016) propose rank mobility measures that are based on two dominant measures of non-parametric rank correlation, namely, Spearman's (1904) $\rho$ index and Kendall's (1938) $\tau$ index. D'Agostino and Dardanoni (2009) characterize rank mobility pre-orders that are linked to Spearman's $\rho$ index; Bossert et al. (2016), on the other hand, focus on mobility measures that have their foundation in Kendall's $\tau$ index. The latter is at the core of the Kemeny distance, which is one of the most prominent distance measures for orderings; see Kemeny (1959) and Kemeny and Snell (1962), where the Kemeny distance is characterized. As pointed out by Can and Storcken (2013), this axiomatization involves a redundant axiom, an observation that allows Can and Storcken (2013) to substantially improve on Kemeny and Snell's (1962) result.

In this paper, we depart from the approach based on measures of rank correlation followed by D'Agostino and Dardanoni (2009) and Bossert et al. (2016). We develop an axiomatic framework from first principles rather than confining ourselves to tools that have their origins in a different (but, of course, closely related) subfield of statistical analysis.

Our central result is a characterization of what we refer to as the head-count ratio. It is a simple and intuitively appealing measure that conveys the basic principles of rank mobility in a transparent manner. The index calculates the rank mobility value by counting the number of individuals who change position in the move from period zero to period one, and divides the resulting value by the size of the population under consideration. The axioms employed in our characterization are plausible and not difficult to justify. We then proceed to a decomposition of the head-count ratio into an upwards head-count ratio and a downwards head-count ratio. As their labels suggest, these measures are obtained by dividing the number of those who move up (respectively, down) by the population size. The benefit of such a decomposition into two opposing measures is the ability to compare the number of individuals who move down with the number of those who move up (at the expense of the former). Our characterizations of these directional mobility measures are achieved by formulating suitable adaptations of the overall mobility axioms to the respective directional case. Again, the resulting properties are easily justifiable and intuitively appealing. We conclude that our new index, along with its directional components, provides an attractive alternative to measures that are currently in use. It has a simple and transparent structure, it is 
straightforward to calculate and to interpret, and it allows policymakers to access the relevant mobility information by means of a tool that is easy to use while resting on a solid theoretical foundation.

The next section introduces the basic notion of rank mobility. Our new rank mobility measure - the head-count ratio - is presented, discussed and characterized in Section II. A natural decomposition into upwards rank mobility and downwards rank mobility, along with the requisite characterization results, follows in Section III. Section III also discusses some conceptual difficulties when considering weighted means of upwards and downwards rank mobility. Section IV concludes, with a discussion of our results and an application of the indices that we propose to the most well-known multidimensional measure of wellbeing and indicator of progress of countries beyond GDP, the Human Development Index.

\section{RANK MOBILITY}

A society is represented by a finite set $N$ of individuals. For our first result, we require that $N$ has at least six members, that is, $n=|N| \geq 6$; for the remaining observations, it is sufficient to assume that $n \geq 2$. We will discuss these cardinality requirements regarding the set $N$ in more detail once we reach the relevant parts of the paper. A strict ordering on $N$ is a complete, transitive and antisymmetric binary relation $R \subseteq N \times N$. The set of all strict orderings on $N$ is denoted by $\mathcal{R}^{N}$. For convenience, we sometimes express strict orderings in line notation, that is, we list the elements of $N$ in decreasing order of rank. For instance, if $N=\{a, b, c\}$, then $R=a b c$ is the strict ordering that ranks $a$ above $b$, and $b$ above $c$. For $a \in N$ and $R \in \mathcal{R}^{N}$, the rank of individual $a$ in $R$ is denoted by $r(a, R)$, that is,

$$
r(a, R)=|\{b \in N: b R a\}| .
$$

Thus for $R=a b c$, we have $r(a, R)=|\{a\}|=1, r(b, R)=|\{a, b\}|=2$ and $r(c, R)=$ $|\{a, b, c\}|=3$.

A rank mobility measure uses as the only relevant information a pair of rankings of the individuals in $N$-one ranking for the previous period, one for the current period. Thus we write such a measure as a function

$$
M: \mathcal{R}^{N} \times \mathcal{R}^{N} \rightarrow[0,1]
$$

where $M\left(R^{0}, R^{1}\right)$ is the mobility associated with a move from the ranking $R^{0}$ in period zero (the previous period) to the ranking $R^{1}$ in period one (the current period). That the range of this measure is given by the interval $[0,1]$ does not involve any loss of generality.

For a pair $\left(R^{0}, R^{1}\right) \in \mathcal{R}^{N} \times \mathcal{R}^{N}$, we denote the set of agents whose ranking improved when moving from period zero to period one by

$$
R^{0} \triangle R^{1}=\left\{a \in N: r\left(a, R^{0}\right)>r\left(a, R^{1}\right)\right\} .
$$

Analogously, the set of agents who dropped in the move from period zero to period one is denoted by

$$
R^{0} \nabla R^{1}=\left\{a \in N: r\left(a, R^{0}\right)<r\left(a, R^{1}\right)\right\} .
$$


The union of these two sets gives us the set of all agents whose ranking changed, and we denote it by

$$
R^{0} \diamond R^{1}=\left\{a \in N: r\left(a, R^{0}\right) \neq r\left(a, R^{1}\right)\right\}
$$

It follows immediately that for any $\left(R^{0}, R^{1}\right) \in \mathcal{R}^{N} \times \mathcal{R}^{N}$,

$$
R^{0} \triangle R^{1}=R^{1} \nabla R^{0} \quad \text { and } \quad R^{0} \diamond R^{1}=R^{1} \diamond R^{0}
$$

That is, $R^{0} \triangle R^{1}$ and $R^{1} \nabla R^{0}$ are in a dual relationship, whereas the set $R^{0} \diamond R^{1}$ has a symmetry property.

For $k \in\{2, \ldots, n\}$, we say that a pair $\left(R^{0}, R^{1}\right) \in \mathcal{R}^{N} \times \mathcal{R}^{N}$ is of degree $k$ if $\left|R^{0} \diamond R^{1}\right|=k$. Analogously, for $k \in\{1, \ldots, n-1\}$, the pair $\left(R^{0}, R^{1}\right) \in \mathcal{R}^{N} \times \mathcal{R}^{N}$ is of upwards (respectively, downwards) degree $k$ if $\left|R^{0} \triangle R^{1}\right|=k$ (respectively, $\left|R^{0} \nabla R^{1}\right|=k$ ).

\section{THE HEAD-COUNT RATIO}

We propose a simple and intuitive measure of rank mobility that is obtained by counting the number of individuals whose positions changed from period zero to period one and dividing the resulting value by the size of the population under consideration. This headcount ratio $M^{H}$ is defined formally by letting, for all $\left(R^{0}, R^{1}\right) \in \mathcal{R}^{N} \times \mathcal{R}^{N}$,

$$
M^{H}\left(R^{0}, R^{1}\right)=\frac{\left|R^{0} \diamond R^{1}\right|}{n}
$$

We emphasize that, as alluded to in the Introduction, this new index has the advantage of being simple and transparent with a natural interpretation. In the remainder of this section, we establish that the measure also has a sound theoretical foundation. We do so by showing that the head-count ratio $M^{H}$ can be characterized by means of four intuitively appealing axioms.

Our first property is anonymity, a standard condition that requires a rank mobility measure to treat all individuals impartially, paying no attention to their identities. Thus we require that permuting the labels that we assign to the members of society does not change the value of a rank mobility measure. Let $\pi: N \rightarrow N$ be a bijective function. For $R \in \mathcal{R}^{N}$, we define the relation $R_{\pi}$ by letting, for all $a, b \in N$,

$$
(\pi(a), \pi(b)) \in R_{\pi} \quad \Longleftrightarrow \quad(a, b) \in R
$$

Thus $\pi$ permutes the labels of the individuals by assigning the label $\pi(a) \in N$ to the individual that was previously labelled $a \in N$.

Anonymity. For all $\left(R^{0}, R^{1}\right) \in \mathcal{R}^{N} \times \mathcal{R}^{N}$ and for all permutations $\pi: N \rightarrow N$,

$$
M\left(R_{\pi}^{0}, R_{\pi}^{1}\right)=M\left(R^{0}, R^{1}\right)
$$

Next, we normalize the maximal possible value of $M$ to the number 1 .

Normalization. $\max \left\{M\left(R^{0}, R^{1}\right):\left(R^{0}, R^{1}\right) \in \mathcal{R}^{N} \times \mathcal{R}^{N}\right\}=1$. 
Normalization ensures that the rank mobility measure is proportional in the sense that it allows for meaningful comparisons of societies with different population sizes. For instance, consider a society with a given number of members and a given pair of rankings. Suppose that this society is to be compared with a society that is composed of twice as many individuals, and there are two moves in the second group for each move in the first - that is, the proportions of those whose positions change are the same in both cases. It is natural to expect that the resulting mobility values are the same. Normalization guarantees that this proportionality property is satisfied. As a consequence, it is clear that the head-count ratio $M^{H}$ is proportional in the abovedescribed sense. In general, if an $n$-person society represented by a pair $\left(R^{0}, R^{1}\right)$ is compared to a society with $m n$ members (where $m \geq 2$ ) in a way such that the number of individuals who change positions in the original pair is multiplied by $m$, then it follows immediately that the value of $M^{H}$ calculated for the second society is

$$
\frac{m\left|R^{0} \diamond R^{1}\right|}{m n}=\frac{\left|R^{0} \diamond R^{1}\right|}{n},
$$

and hence the proportionality property is satisfied. See Bossert et al. (2016) for a related discussion that concerns replication-invariant rank mobility measures in a variable population setting.

To reflect the feature that our measure depends only on those individuals who experience a change in their respective rank, we employ an invariance axiom. We require the rank mobility values associated with two pairs of strict orderings $\left(R^{0}, R^{1}\right),\left(\bar{R}^{0}, \bar{R}^{1}\right) \in \mathcal{R}^{N} \times \mathcal{R}^{N}$ to coincide whenever the sets of individuals whose position changes are the same.

Change invariance. For all $\left(R^{0}, R^{1}\right),\left(\bar{R}^{0}, \bar{R}^{1}\right) \in \mathcal{R}^{N} \times \mathcal{R}^{N}$, if $R^{0} \diamond R^{1}=\bar{R}^{0} \diamond \bar{R}^{1}$, then

$$
M\left(R^{0}, R^{1}\right)=M\left(\bar{R}^{0}, \bar{R}^{1}\right)
$$

Finally, we introduce an additivity property with an intuitive interpretation. Consider a situation in which the rank mobility associated with a move from period zero to period one is determined and, analogously, the rank mobility corresponding to a move from period one to period two is calculated (we can think of the time periods under consideration as years, for instance). Now suppose that we want to measure the mobility associated with the move from period zero to period two (that is, consider a biannual setting). If the set of individuals whose rank changed in the move from period zero to period one and the set of those whose rank changed in the move from period one to period two are disjoint, then it seems natural to combine these distinct (annual) moves in an additive manner (as a biannual move). That is, if it so happens that the rank changes from period zero to period one and from period one to period two are distinct in the sense that they involve different individuals, then the rank mobility associated with a move from period zero to period two is given by the sum of the rank mobility for the two moves from zero to one and from one to two. Thus we impose the following property.

Change additivity. For all $R^{0}, R^{1}, R^{2} \in \mathcal{R}^{N}$, if $\left(R^{0} \diamond R^{1}\right) \cap\left(R^{1} \diamond R^{2}\right)=\varnothing$, then

$$
M\left(R^{0}, R^{2}\right)=M\left(R^{0}, R^{1}\right)+M\left(R^{1}, R^{2}\right) .
$$


We can now state and prove our first characterization result. As will become clear once we go through the proof, the result is valid only for societies with at least six members.

Theorem 1. Given any $N$ with $n \geq 6$, a rank mobility measure $M$ satisfies anonymity, normalization, change invariance and change additivity if and only if $M=M^{H}$.

Proof. It is straightforward to verify that $M^{H}$ satisfies the axioms of the theorem statement. Now suppose that $M$ is a rank mobility measure that satisfies the axioms. Let $k \in\{2, \ldots, n\}$, and consider any pair $\left(R^{0}, R^{1}\right) \in \mathcal{R}^{N} \times \mathcal{R}^{N}$ that is of degree $k$, that is, $\left|R^{0} \diamond R^{1}\right|=k$. Let $N^{\prime}=R^{0} \diamond R^{1}$, and define $\omega_{N^{\prime}}^{k}=M\left(R^{0}, R^{1}\right)$. Now consider any $\bar{N} \subseteq N$ and $\left(\bar{R}^{0}, \bar{R}^{1}\right) \in \mathcal{R}^{N} \times \mathcal{R}^{N}$ such that

$$
\left|\bar{R}^{0} \diamond \bar{R}^{1}\right|=|\bar{N}|=\left|N^{\prime}\right|=k
$$

If $\bar{N}=N^{\prime}$, then $M\left(\bar{R}^{0}, \bar{R}^{1}\right)=M\left(R^{0}, R^{1}\right)=\omega_{N^{\prime}}^{k}$ follows from change invariance.

If $\bar{N} \neq N^{\prime}$, then there exists a permutation $\pi: N \rightarrow N$ such that

$$
\left(R_{\pi}^{0}, R_{\pi}^{1}\right)=\left(\bar{R}^{0}, \bar{R}^{1}\right) \text { and } \bar{N}=\left\{\pi(a): a \in N^{\prime}\right\} .
$$

By anonymity,

$$
\omega \frac{k}{N}=M\left(\bar{R}^{0}, \bar{R}^{1}\right)=M\left(R_{\pi}^{0}, R_{\pi}^{1}\right)=M\left(R^{0}, R^{1}\right)=\omega_{N^{\prime}}^{k}
$$

and because $\bar{N}$ can be any arbitrary subset of $N$ with $k$ members, it follows that $\omega_{\frac{k}{N}}^{k}=\omega_{N^{\prime}}^{k}$ for all pairs of degree $k$. Thus $\omega_{N^{\prime}}^{k}$ cannot depend on $N^{\prime}$, and we write it as $\omega^{k}$. It therefore follows that $M\left(R^{0}, R^{1}\right)=\omega^{k}$ for all pairs $\left(R^{0}, R^{1}\right)$ of degree $k$.

Next, we establish a relationship between the $\omega^{k}$ values. Let $\left(R^{0}, R^{1}\right) \in \mathcal{R}^{N} \times \mathcal{R}^{N}$ be of degree $k$. Without loss of generality, suppose that $R^{0} \diamond R^{1}=\left\{a_{1}, \ldots, a_{k}\right\}$.

Case $1: k$ is even. Let $\left(\bar{R}^{0}, \bar{R}^{1}\right) \in \mathcal{R}^{N} \times \mathcal{R}^{N}$ be such that $\bar{R}^{0} \diamond \bar{R}^{1}=R^{0} \diamond R^{1}$ $=\left\{a_{1}, \ldots, a_{k}\right\}$ and

$$
\begin{aligned}
& \bar{R}^{0}=\left(a_{1} a_{2}\right)\left(a_{3} a_{4}\right) \ldots\left(a_{k-1} a_{k}\right) \ldots, \\
& \bar{R}^{1}=\left(a_{2} a_{1}\right)\left(a_{4} a_{3}\right) \ldots\left(a_{k} a_{k-1}\right) \ldots,
\end{aligned}
$$

where we use the parentheses to emphasize the individuals who change positions when moving from $\bar{R}^{0}$ to $\bar{R}^{1}$. By change invariance, it follows that $M\left(R^{0}, R^{1}\right)=M\left(\bar{R}^{0}, \bar{R}^{1}\right)=\omega^{k}$ because $\left(\bar{R}^{0}, \bar{R}^{1}\right)$ is of degree $k$. The move from $\bar{R}^{0}$ to $\bar{R}^{1}$ can be decomposed into $k / 2$ pairs of degree 2 , where the sets of individuals whose positions change in each of these moves are given by $\left\{a_{1}, a_{2}\right\}, \ldots,\left\{a_{k-1}, a_{k}\right\}$. These sets are pairwise disjoint, and change additivity can be applied repeatedly to conclude that 


$$
M\left(R^{0}, R^{1}\right)=M\left(\bar{R}^{0}, \bar{R}^{1}\right)=\omega^{k}=\frac{k}{2} \omega^{2}
$$

for all even $k$.

Case $2: k$ is odd. Let $\left(\bar{R}^{0}, \bar{R}^{1}\right) \in \mathcal{R}^{N} \times \mathcal{R}^{N}$ be such that $\bar{R}^{0} \diamond \bar{R}^{1}=R^{0} \diamond R^{1}=$ $\left\{a_{1}, \ldots, a_{k}\right\}$ and

$$
\begin{aligned}
& \bar{R}^{0}=\left(a_{1} a_{2}\right)\left(a_{3} a_{4}\right) \ldots\left(a_{k-4} a_{k-3}\right)\left(a_{k-2} a_{k-1} a_{k}\right) \ldots \\
& \bar{R}^{1}=\left(a_{2} a_{1}\right)\left(a_{4} a_{3}\right) \ldots\left(a_{k-3} a_{k-4}\right)\left(a_{k} a_{k-2} a_{k-1}\right) \ldots
\end{aligned}
$$

By change invariance, it follows that $M\left(R^{0}, R^{1}\right)=M\left(\bar{R}^{0}, \bar{R}^{1}\right)=\omega^{k}$ because $\left(\bar{R}^{0}, \bar{R}^{1}\right)$ is of degree $k$. The move from $\bar{R}^{0}$ to $\bar{R}^{1}$ can be decomposed into $(k-3) / 2$ pairs of degree 2 and one pair of degree 3 . The sets of individuals whose positions change in each of these moves are $\left\{a_{1}, a_{2}\right\}, \ldots,\left\{a_{k-4}, a_{k-3}\right\}$ and $\left\{a_{k-2}, a_{k-1}, a_{k}\right\}$. Again, these sets are pairwise disjoint, and invoking change additivity repeatedly, it follows that

$$
M\left(R^{0}, R^{1}\right)=M\left(\bar{R}^{0}, \bar{R}^{1}\right)=\omega^{k}=\frac{k-3}{2} \omega^{2}+\omega^{3}
$$

for all odd $k$.

Because $n \geq 6$, there exist $\left(R^{0}, R^{1}\right),\left(\bar{R}^{0}, \bar{R}^{1}\right) \in \mathcal{R}^{N} \times \mathcal{R}^{N}$ such that

$$
R^{0} \diamond R^{1}=\bar{R}^{0} \diamond \bar{R}^{1}=\left\{a_{1}, a_{2}, a_{3}, a_{4}, a_{5}, a_{6}\right\}
$$

so both of these pairs are of degree $k=6$, and

$$
\begin{aligned}
R^{0} & =\left(a_{1} a_{2}\right)\left(a_{3} a_{4}\right)\left(a_{5} a_{6}\right) \ldots, \\
R^{1} & =\left(a_{2} a_{1}\right)\left(a_{4} a_{3}\right)\left(a_{6} a_{5}\right) \ldots, \\
\bar{R}^{0}=R^{0} & =\left(a_{1} a_{2} a_{3}\right)\left(a_{4} a_{5} a_{6}\right) \ldots, \\
\bar{R}^{1} & =\left(a_{2} a_{3} a_{1}\right)\left(a_{5} a_{6} a_{4}\right) \ldots ;
\end{aligned}
$$

again, parentheses are used to emphasize the positional changes. By change invariance, it follows that $M\left(R^{0}, R^{1}\right)=M\left(\bar{R}^{0}, \bar{R}^{1}\right)=\omega^{6}$. Using change additivity, we obtain

$$
M\left(R^{0}, R^{1}\right)=\omega^{6}=3 \omega^{2}
$$

and

$$
M\left(\bar{R}^{0}, \bar{R}^{1}\right)=\omega^{6}=2 \omega^{3} .
$$


Thus $3 \omega^{2}=2 \omega^{3}$, which implies

$$
\omega^{3}=\frac{3}{2} \omega^{2}
$$

Substituting into (2), we obtain

$$
\omega^{k}=\frac{k-3}{2} \omega^{2}+\frac{3}{2} \omega^{2}=\frac{k}{2} \omega^{2}
$$

for all odd $k$. Thus (1) is true for all (odd and even) $k \in\{2, \ldots, n\}$.

By normalization, there exists a pair $\left(R^{0}, R^{1}\right) \in \mathcal{R}^{N} \times \mathcal{R}^{N}$ such that $M$ assumes its maximal value of 1 at $\left(R^{0}, R^{1}\right)$. Because $\omega^{k}$ is increasing in $k$, this maximal value is obtained for $k=n$. Using (1), it follows that $\omega^{n}=(n / 2) \omega^{2}=1$ and hence $\omega^{2}=2 / n$. Thus for any $\left(R^{0}, R^{1}\right) \in \mathcal{R}^{N} \times \mathcal{R}^{N}$ of degree $k$, it follows that

$$
M\left(R^{0}, R^{1}\right)=\omega^{k}=\frac{k}{2} \frac{2}{n}=\frac{\left|R^{0} \diamond R^{1}\right|}{n}=M^{H}\left(R^{0}, R^{1}\right) . \text { Q.E.D. }
$$

The assumption that $N$ consists of at least six members is essential in the above proof. The $\omega^{k}$ values are determined by considering multiples of degree-two situations and degree-three situations, and the combination of the two yields the desired values. But this method can be applied only if there are at least six individuals to begin with: six is the smallest number that can be expressed as a multiple of two and as a multiple of three. It is straightforward to see that if $N$ has fewer than six members, then other measures become available, and thus this minimal-cardinality assumption cannot be dispensed with in the above theorem.

\section{UPWARDS AND DOWNWARDS MOBILITY RATIOS}

Suppose now that we want to focus on upwards rank mobility, that is, we pay particular attention to individuals who move up in the ranking. A natural modification of the headcount ratio $M^{H}$ is obtained if the number of those who change position is replaced by the number of those who move up in the ranking, again divided by the total number of agents. Thus we define the upwards head-count ratio $M^{U}$ by letting, for all $\left(R^{0}, R^{1}\right) \in \mathcal{R}^{N} \times \mathcal{R}^{N}$,

$$
M^{U}\left(R^{0}, R^{1}\right)=\frac{\left|R^{0} \triangle R^{1}\right|}{n} .
$$

Note that $M^{U}$ does not satisfy the normalization axiom employed in our characterization of $M^{H}$. This is because imposing a maximal value of 1 on a directional measure would generate a conflict with the proportionality principle. Indeed, if we were to assume that upwards rank mobility achieves a maximal value of 1 , then the characterization below would yield the ratio 


$$
\frac{\left|R^{0} \triangle R^{1}\right|}{n-1}
$$

This is the case because the minimal positive number of upwards changes is 1 and the maximal number of upwards changes is $n-1$ : if someone moves up in the ranking, then at least one other person must move down. It is immediate that the measure of (3) is not proportional. An $m$-fold multiple of an $n$-person society leads to the ratio

$$
\frac{m\left|R^{0} \triangle R^{1}\right|}{m n-1} .
$$

Clearly, this ratio is not equal to that of (3). In contrast, the upwards head-count ratio $M^{U}$ obviously is proportional. For this reason, the normalization axiom used in Theorem 1 needs to be modified in order to arrive at $M^{U}$. To ensure proportionality, the maximal value achieved by a measure of upwards rank mobility must be equal to $(n-1) / n$ rather than 1 . This alternative normalization is very plausible because upwards rank mobility cannot but yield merely a partial picture of overall mobility. Thus we obtain the following directional variant of the normalization property.

Directional normalization. $\max \left\{M\left(R^{0}, R^{1}\right):\left(R^{0}, R^{1}\right) \in \mathcal{R}^{N} \times \mathcal{R}^{N}\right\}=(n-1) / n$.

Analogously, we can define a downwards head-count ratio $M^{D}$ by concentrating on downwards moves. That is, for all $\left(R^{0}, R^{1}\right) \in \mathcal{R}^{N} \times \mathcal{R}^{N}$,

$$
M^{D}\left(R^{0}, R^{1}\right)=\frac{\left|R^{0} \nabla R^{1}\right|}{n}
$$

Again, we divide by $n$ so that the resulting measure is proportional.

It is now immediate that the head-count ratio $M^{H}$ can be decomposed naturally into its upwards and downwards constituent parts by adding $M^{U}$ and $M^{D}$. That is, we have, for all $\left(R^{0}, R^{1}\right) \in \mathcal{R}^{N} \times \mathcal{R}^{N}$,

$$
M^{H}\left(R^{0}, R^{1}\right)=M^{U}\left(R^{0}, R^{1}\right)+M^{D}\left(R^{0}, R^{1}\right) .
$$

We now provide characterizations of $M^{U}$ and of $M^{D}$. To do so, the two axioms of change invariance and change additivity can be rephrased in a natural way to capture upwards and downwards rank mobility. Instead of focusing on the set of agents whose positions change in the move from $R^{0}$ to $R^{1}$, we restrict attention to those whose positions improve (respectively, worsen) so that we obtain the following properties.

Upwards invariance. For all $\left(R^{0}, R^{1}\right),\left(\bar{R}^{0}, \bar{R}^{1}\right) \in \mathcal{R}^{N} \times \mathcal{R}^{N}$, if $R^{0} \triangle R^{1}=\bar{R}^{0} \triangle \bar{R}^{1}$, then

$$
M\left(R^{0}, R^{1}\right)=M\left(\bar{R}^{0}, \bar{R}^{1}\right)
$$

Downwards invariance. For all $\left(R^{0}, R^{1}\right),\left(\bar{R}^{0}, \bar{R}^{1}\right) \in \mathcal{R}^{N} \times \mathcal{R}^{N}$, if $R^{0} \nabla R^{1}=\bar{R}^{0} \nabla \bar{R}^{1}$, then

$$
M\left(R^{0}, R^{1}\right)=M\left(\bar{R}^{0}, \bar{R}^{1}\right)
$$


Upwards additivity. For all $R^{0}, R^{1}, R^{2} \in \mathcal{R}^{N}$, if $\left(R^{0} \triangle R^{1}\right) \cap\left(R^{1} \triangle R^{2}\right)=\varnothing$, then

$$
M\left(R^{0}, R^{2}\right)=M\left(R^{0}, R^{1}\right)+M\left(R^{1}, R^{2}\right) .
$$

Downwards additivity. For all $R^{0}, R^{1}, R^{2} \in \mathcal{R}^{N}$, if $\left(R^{0} \nabla R^{1}\right) \cap\left(R^{1} \nabla R^{2}\right)=\varnothing$, then

$$
M\left(R^{0}, R^{2}\right)=M\left(R^{0}, R^{1}\right)+M\left(R^{1}, R^{2}\right) .
$$

The property of anonymity need not be changed; it is just as natural and plausible in the directional case. We can now state and prove two theorems that parallel Theorem 1. However, their proofs differ somewhat from that of our first theorem. In particular, it is now no longer necessary to require that there be at least six agents - any society with at least two members is covered by these results.

Theorem 2. Given any $N$ with $n \geq 2$, a rank mobility measure $M$ satisfies anonymity, directional normalization, upwards invariance and upwards additivity if and only if $M=M^{U}$.

Proof. Again, it is straightforward to verify that $M^{U}$ satisfies all of the axioms. Now suppose that $M$ is a rank mobility measure that satisfies the four axioms. Let $k \in\{1, \ldots, n-1\}$ and consider any pair $\left(R^{0}, R^{1}\right) \in \mathcal{R}^{N} \times \mathcal{R}^{N}$ that is of upwards degree $k$, that is, $\left|R^{0} \triangle R^{1}\right|=k$. Replacing change invariance with upwards invariance and $R^{0} \diamond R^{1}$ with $R^{0} \triangle R^{1}$ in the corresponding part of the proof of Theorem 1, we conclude that there exists $\omega^{k}$ such that $M\left(R^{0}, R^{1}\right)=\omega^{k}$ for all pairs $\left(R^{0}, R^{1}\right) \in \mathcal{R}^{N} \times \mathcal{R}^{N}$ that are of upwards degree $k$.

Now consider the following three rankings:

$$
\begin{aligned}
& R^{0}=a_{k+1} a_{1} a_{2} a_{3} \ldots a_{k-2} a_{k-1} a_{k} \ldots, \\
& R^{1}=a_{1} a_{2} a_{3} \ldots a_{k-2} a_{k-1} a_{k+1} a_{k} \ldots, \\
& R^{2}=a_{1} a_{2} a_{3} \ldots a_{k-2} a_{k-1} a_{k} a_{k+1} \ldots .
\end{aligned}
$$

Note that $R^{0} \triangle R^{1}=\left\{a_{1}, \ldots, a_{k-1}\right\}$ and $R^{1} \triangle R^{2}=\left\{a_{k}\right\}$. Therefore $\left(R^{0}, R^{1}\right)$ is of degree $k-1$, and $\left(R^{1}, R^{2}\right)$ is of degree 1 . Moreover, the two sets are disjoint and we can employ upwards additivity to obtain

$$
\omega^{k}=M\left(R^{0}, R^{2}\right)=M\left(R^{0}, R^{1}\right)+M\left(R^{1}, R^{2}\right)=\omega^{k-1}+\omega^{1},
$$

and a simple iteration argument yields

$$
\omega^{k}=k \omega^{1}
$$

for all $k \in\{1, \ldots, n-1\}$. Because $\omega^{k}$ is increasing in $k$, it follows that its maximal value is achieved at pairs of degree $n-1$. Thus, by normalization, $\omega^{n-1}=(n-1) \omega^{1}=(n-1) / n$ and hence

$$
\omega^{1}=\frac{1}{n}
$$


Because $M\left(R^{0}, R^{1}\right)=\omega^{k}$ for all pairs $\left(R^{0}, R^{1}\right) \in \mathcal{R}^{N} \times \mathcal{R}^{N}$ that are of upwards degree $k$, it follows that

$$
M\left(R^{0}, R^{1}\right)=\frac{k}{n}=\frac{\left|R^{0} \triangle R^{1}\right|}{n}=M^{U}\left(R^{0}, R^{1}\right) . \text { Q.E.D. }
$$

Clearly, the above proof works in the same way if upwards invariance and upwards additivity are replaced with downwards invariance and downwards additivity. Thus we immediately obtain the following characterization of our measure of downwards rank mobility $M^{D}$.

Theorem 3. Given any $N$ with $n \geq 2$, a rank mobility measure $M$ satisfies anonymity, directional normalization, downwards invariance and downwards additivity if and only if $M=M^{D}$.

In some applications, one may want to attach different weights to upwards and downwards moves, thus expressing overall rank mobility as a weighted mean of $M^{U}$ and $M^{D}$. Letting $\alpha \in[0,1]$ denote the weight attached to upwards mobility, the corresponding measure can be expressed as

$$
M^{\alpha}\left(R^{0}, R^{1}\right)=\alpha M^{U}\left(R^{0}, R^{1}\right)+(1-\alpha) M^{D}\left(R^{0}, R^{1}\right)
$$

for all $\left(R^{0}, R^{1}\right) \in \mathcal{R}^{N} \times \mathcal{R}^{N}$. The interpretation of (4) it straightforward: the higher the value of $\alpha$, the higher the relative importance of upwards movements. In general, the maximal value of $M^{\alpha}$ is

$$
M_{\max }^{\alpha}= \begin{cases}(1-\alpha)+\alpha /(n-1) & \text { if } \alpha \in[0,1 / 2] \\ \alpha+(1-\alpha) /(n-1) & \text { if } \alpha \in(1 / 2,1] .\end{cases}
$$

This observation raises an immediate concern when it comes to the choice of a normalization property. Because these maximal values are $\alpha$-specific, it is far from obvious how a suitable normalization axiom may be formulated. Although seemingly appealing at first sight, there appears to be a conceptual problem with calculating weighted means of the directional measures. Because the measures themselves have a strong ordinal flavour, it is not an easy task to endow these weighted means with a solid interpretation. For example, does a weight of $1 / 4$ accurately capture the idea that downwards mobility is three times as important as upwards mobility? Based on these considerations, it seems plausible to us that calculating weighted means may take us well beyond the ordinal nature of the measures studied here.

\section{DISCUSSION AND AN EMPIRICAL APPLICATION}

The measures proposed in this paper are simple and intuitive indices of rank mobility based on the count of individuals or countries who change their position from one period to the next. However, our approach can serve as the basis of more complex aggregation procedures. For example, one may want to capture the intensity of period-to-period 
movements by incorporating information on the number of positions involved in each individual change. Another interesting aspect of mobility not explored here is the likelihood of expected changes, and weighted counts may be performed, where different weights are given to changes occurring in different positions of the ranking.

An interesting statistic derived from our measures is the ratio of upwards and downwards mobility ratios, that is, the ratio

$$
\frac{M^{U}\left(R^{0}, R^{1}\right)}{M^{D}\left(R^{0}, R^{1}\right)}=\frac{\left|R^{0} \triangle R^{1}\right|}{\left|R^{0} \nabla R^{1}\right|}
$$

(provided that the number of those who move is not equal to zero). This ratio is a useful indicator of improvement relative to deterioration in the move from one period to the next.

From an applied perspective, note that the proposed measures are defined on strict rankings. But ties occur frequently in data such as those relating to household incomes, for instance. The measure of income that is usually attributed to individuals is equivalent household income, thus all household members have the same income level and hence the same rank. Of course, this issue can be dealt with by considering households to be the relevant units rather than individuals. An additional potential problem arises in selfreported income data: individuals tend to report rounded values such as $\$ 3500$ rather than $\$ 3473.84$, say. The resulting clustering gives rise to further ties in the ranking. One way in which our measures can be applied in such cases is to consider all possible ways of breaking ties in these rankings, then calculate the index values for each of the resulting strict rankings, and finally perform an averaging operation.

Ties in rankings are unlikely to occur in many settings where the mobility of groups or countries is considered. The average income of a group (such as teachers, physicians, and so on) can be used as a proxy of the social status of these groups in a society. Analogously, countries are frequently ranked according to GDP per capita or to indices beyond GDP such as the Human Development Index or the Better Life Index - and ties are extremely rare in these contexts. What matters in public debates on these issues is the relative ranking of countries derived from these indices rather than the values of the indicators per se. Thus this is another area in which our results can be applied successfully and to which we turn next.

The remainder of the paper is devoted to an application of the proposed measure to the first and most well-known multidimensional measure of wellbeing: the Human Development Index (henceforth HDI), introduced in the first Human Development Report (henceforth HDR) by the United Nations Development Programme in 1990. The HDI is an extremely popular measure among policymakers and the general public because of its intuitive character and the large number of countries entering the evaluation. It measures human development with the three key dimensions of leading a long and healthy life, being knowledgeable, and having a decent standard of living.

The current version of the HDI was introduced on the occasion of the twentieth anniversary of the HDR in 2010. According to the definition available in United Nations Development Programme (2017a):

The health dimension is assessed by life expectancy at birth, the education dimension is measured by mean of years of schooling for adults aged 25 years and more and expected years of schooling for children of school entering age. The standard of living dimension is measured by 
gross national income [GNI] per capita. The HDI uses the logarithm of income, to reflect the diminishing importance of income with increasing GNI. The scores for the three HDI dimension indices are then aggregated into a composite index using [the] geometric mean.

To maximize the number of countries included in our study, we focus on the years between 2010 and 2015, the latter being the most recent available year at the time of our analysis. Before 2014, data for Sudan include South Sudan, which renders the analysis for the two countries inconsistent, and for this reason we exclude both. See United Nations Development Programme (2017b) for the list of included countries. It is standard practice in the HDR to report values of the HDI only up to the third decimal place, accepting a few ties in the ranking. When no such approximation is performed, ties are very unlikely, and indeed we do not observe any two countries among the 186 considered with the same annual HDI. We compute the head-count ratio $M^{H}$ along with its two directional components $M^{U}$ and $M^{D}$ for yearly mobility profiles. Moreover, the values of the ratio of upwards and downwards mobility are included because this statistic provides a clear and intuitive indication of positional improvements versus deteriorations.

The results, summarized in Table 1, are presented for the entire set of 186 countries but also separately for the 35 countries belonging to the OECD and the remaining 151 who are not members of this entity. The head-count ratio oscillates over the period under consideration, reaching its minimum value of 0.785 in the move from 2014 to 2015 . When we distinguish between OECD and non-OECD countries, we notice that the head-count ratio for the former decreases over time from its highest value of 0.943 to its lowest level of 0.686 in the period between 2013 and 2014, to move back up to 0.80 in the move from 2014 to 2015. The picture for the non-OECD countries, the great majority of the observations, mimics the path of the entire sample where we observe an increase in persistence in the positions from 2012 onwards. Among the countries primarily responsible for the overall decrease in the head-count ratio are the Czech Republic

TABLE 1

The Head-count Measure of Rank Mobility for the Human Development IndeX FOR 186 COUNTRIES OVER THE PERIOD 2010-15

\begin{tabular}{lccccc}
\hline & $2010-11$ & $2011-12$ & $2012-13$ & $2013-14$ & $2014-15$ \\
\hline Head-count & & & & & \\
Entire sample & 0.919 & 0.806 & 0.898 & 0.849 & 0.785 \\
Non-OECD & 0.914 & 0.801 & 0.921 & 0.887 & 0.781 \\
OECD & 0.943 & 0.829 & 0.800 & 0.686 & 0.800 \\
Upwards & & & & & \\
Entire sample & 0.495 & 0.382 & 0.371 & 0.398 & 0.382 \\
Non-OECD & 0.503 & 0.364 & 0.391 & 0.430 & 0.384 \\
OECD & 0.457 & 0.457 & 0.286 & 0.257 & 0.371 \\
Downwards & & & & & \\
Entire sample & 0.425 & 0.425 & 0.527 & 0.452 & 0.403 \\
Non-OECD & 0.411 & 0.437 & 0.530 & 0.457 & 0.397 \\
OECD & 0.486 & 0.371 & 0.514 & 0.429 & 0.429 \\
Upwards/downwards ratio & 1.165 & 0.899 & 0.704 & 0.881 & 0.947 \\
Entire sample & 1.226 & 0.833 & 0.738 & 0.942 & 0.967 \\
Non-OECD & 0.941 & 1.231 & 0.556 & 0.600 & 0.867 \\
OECD & & & & & \\
\hline
\end{tabular}

Economica

(C) 2017 The London School of Economics and Political Science 
(which remains in the same position starting in 2012), Côte d'Ivoire, Greece (in the same position since 2011), Norway (in the same position since 2011), Palau, Poland and Slovenia (in the same position since 2013).

The upwards head-count ratio reaches its highest value during the first two years and then oscillates around 0.38 . The declining trend in the move towards better positions is especially due to the OECD countries. The downwards head-count ratio for the entire sample and the non-OECD countries follows a reversed-V path, while for the OECD member states the picture more resembles a W. In all cases, $M^{D}$ reaches its highest value for the move from 2012 to 2013.

The last three rows of Table 1 report the values of the ratio of upwards and downwards mobility. Values greater than 1 indicate that there are relatively more countries whose human development is improving, which is a result that we observe only once and in different years: for the entire sample and for the non-OECD countries in the move from 2010 to 2011, and for the OECD countries in the transition from 2011 to 2012. For the entire sample and the non-OECD countries, the ratio is U-shaped over time, with the lowest value of 0.704 (respectively, 0.738 ) reached in the period between 2012 and 2013. For the OECD member states, the peak observed in the change from 2011 to 2012 is followed by a trough that reaches a value as low as 0.556 between 2012 and 2013, indicating that there are almost twice as many OECD countries whose ranks deteriorate as compared to those who move up. This observation provides some cause for concern among international bodies: the countries that move downwards significantly outnumber those that move up, a feature that appears to be rather undesirable. Note that, on the other hand, the requisite ratios increased in each of the two most recent moves. If this is the beginning of an upwards trend, then the international community may be on the right track; it may, however, be too soon to come to an overly optimistic conclusion, and continued close monitoring of these developments seems to be called for.

\section{ACKNOWLEDGMENTS}

We thank the editor Stephen Machin and two anonymous referees for comments. Financial support from the Fonds de Recherche sur la Société et la Culture of Québec, the Social Sciences and Humanities Research Council of Canada, the Netherlands Organisation for Scientific Research (NWO) under the grants Open Competitie (OC: 400-09-354) and Innovational Research Incentives Scheme (VENI 2013: 451-13-017), and the Fonds National de la Recherche Luxembourg, is gratefully acknowledged.

\section{REFERENCES}

Bossert, W., CAN, B. and D’Ambrosio, C. (2016). Measuring rank mobility with variable population size. Social Choice and Welfare, 46, 917-31.

CAn, B. and Storcken, T. (2013). A re-characterization of the Kemeny distance. Maastricht University School of Business and Economics, RM/13/009.

Chetty, R., Hendren, N., Kline, P. and Saez, E. (2014). Where is the land of opportunity? The geography of intergenerational mobility in the United States. Quarterly Journal of Economics, 129, 1553-623.

Cowell, F. A. and Flachaire, E. (2011). Measuring mobility. GREQAM Working Paper no. 2011-21.

D'Agostino, M. and Dardanoni, V. (2009). The measurement of rank mobility. Journal of Economic Theory, 144, 1783-803.

Dickens, R. (1999). Caught in a trap? Wage mobility in Great Britain: 1975-1994. Economica, 67, 477-97.

Fields, G. S. (2008). Income mobility. In S. N. Durlauf and L. E. Blume (eds), The New Palgrave Dictionary of Economics Online. New York: Palgrave Macmillan; available online at www.dictionaryofeconomics.com/ article?id=pde2008_I000271 (accessed 8 October 2017). 
and OK, E. A. (1999). The measurement of income mobility: an introduction to the literature. In J. SILBER (ed.), Handbook of Income Inequality Measurement. Dordrecht: Kluwer Academic Publishers, pp. 557-96.

JÄntti, M. and Jenkins, S. P. (2014). Income mobility. In A. B. AtKInSON and F. BourguignON (eds), Handbook of Income Distribution, Vol. 2. Amsterdam: Elsevier, pp. 807-936.

KeMENy, J. G. (1959). Mathematics without numbers. Daedalus, 88, 577-91.

__ and SNELL, J. L. (1962). Preference rankings: an axiomatic approach. In J. G. KemENY and J. L. SNELL (eds), Mathematical Models in the Social Sciences. New York: Blaisdell Publishing Company, pp. 9-23.

Kendall, M. G. (1938). A new measure of rank correlation. Biometrika, 30, 81-93.

MaAsoumi, E. (1998). On mobility. In D. GILES and A. UllaH (eds), Handbook of Applied Economic Statistics. New York: Marcel Dekker, pp. 119-75.

Nybom, M. and Stuhler, J. (2017). Biases in standard measures of intergenerational income dependence. Journal of Human Resources, 52, 800-25.

SpeARman, C. (1904). The proof and measurement of association between two things. American Journal of Psychology, 15, 72-101.

United Nations Development Programme (2017a). Human Development Index (HDI); available online at http://hdr.undp.org/en/content/human-development-index-hdi (accessed 8 October 2017).

- (2017b). Human Development Data (1990-2015); available online at http://hdr.undp.org/en/data (accessed 8 October 2017).

Venator, J. and Reeves, R. V. (2015). Measuring relative mobility, Part 1. Brookings Social Mobility Memo, 27 April; available online at https://www.brookings.edu/blog/social-mobility-memos/2015/04/27/ measuring-relative-mobility-part-1 (accessed 8 October 2017). 